\title{
Oscillation Conditions for Difference Equations with a Monotone or Nonmonotone Argument
}

\author{
G. M. Moremedi ${ }^{1}$ and I. P. Stavroulakis $\mathbb{I D}^{1,2}$ \\ ${ }^{1}$ Department of Mathematical Sciences, University of South Africa, Pretoria 0003, South Africa \\ ${ }^{2}$ Department of Mathematics, University of Ioannina, 45110 Ioannina, Greece \\ Correspondence should be addressed to I. P. Stavroulakis; ipstav@uoi.gr
}

Received 5 September 2017; Accepted 5 April 2018; Published 21 June 2018

Academic Editor: Manuel De la Sen

Copyright (C) 2018 G. M. Moremedi and I. P. Stavroulakis. This is an open access article distributed under the Creative Commons Attribution License, which permits unrestricted use, distribution, and reproduction in any medium, provided the original work is properly cited.

\begin{abstract}
Consider the first-order delay difference equation with a constant argument $\Delta x(n)+p(n) x(n-k)=0, n=0,1,2, \ldots$, and the delay difference equation with a variable argument $\Delta x(n)+p(n) x(\tau(n))=0, n=0,1,2, \ldots$, where $p(n)$ is a sequence of nonnegative real numbers, $k$ is a positive integer, $\Delta x(n)=x(n+1)-x(n)$, and $\tau(n)$ is a sequence of integers such that $\tau(n) \leq n-1$ for all $n \geq 0$ and $\lim _{n \rightarrow \infty} \tau(n)=\infty$. A survey on the oscillation of all solutions to these equations is presented. Examples illustrating the results are given.
\end{abstract}

\section{Introduction}

In the last few decades the oscillation theory of delay differential equations has been extensively developed. The oscillation theory of discrete analogues of delay differential equations has also attracted growing attention in the recent few years. The reader is referred to [1-36] and the references cited therein. In particular, the problem of establishing sufficient conditions for the oscillation of all solutions of the delay difference equation with a constant argument

$$
\Delta x(n)+p(n) x(n-k)=0, \quad n=0,1,2, \ldots,
$$

and the delay difference equation with a variable argument

$$
\Delta x(n)+p(n) x(\tau(n))=0, \quad n=0,1,2, \ldots,
$$

where $p(n)$ is a sequence of nonnegative real numbers, $k$ is a positive integer, $\Delta x(n)=x(n+1)-x(n)$, and $\tau(n)$ is a sequence of integers such that $\tau(n) \leq n-1$ for all $n \geq 0$ and $\lim _{n \rightarrow \infty} \tau(n)=\infty$, has been the subject of many recent investigations. Strong interest in (1) and (1) ${ }^{\prime}$ is motivated by the fact that they represent the discrete analogues of the delay differential equations (see $[22,37,38]$ and the references cited therein):

$$
\begin{aligned}
& x^{\prime}(t)+p(t) x(t-\tau)=0, \quad p(t) \geq 0, \tau>0, \\
& x^{\prime}(t)+p(t) x(\tau(t))=0, \quad p(t) \geq 0, \tau(t)<t .
\end{aligned}
$$

Set $w=-\min _{n \geq 0} \tau(n)$. Clearly, $w$ is a finite positive integer. By a solution of (1) $\left[(1)^{\prime}\right]$ we mean a sequence $x(n)$ which is defined for $n \geq-k[n \geq-w]$ and which satisfies (1) [(1)'] for $n \geq 0$.

A solution $x(n)$ is said to be oscillatory if the terms $x(n)$ of the solution are not eventually positive or eventually negative. Otherwise, the solution is called nonoscillatory.

For convenience, we will assume that inequalities about values of sequences are satisfied eventually for all large $n$.

For the general theory of these equations, the reader is referred to $[1-3,22,39]$.

Besides the purely mathematical problem, the interest in the behavior of the solutions to difference equations with retarded arguments is justified by the fact that the mathematical modeling of many real-world problems leads to difference equations where the unknown function depends on the past history rather than only the present state. This interest grows stronger as difference equations naturally arise from 
discretization of differential equations. As a consequence, many researchers have been concerned with the study of qualitative behavior of solutions to difference equations, in particular, the study of oscillation of solutions.

In 1969 and in 1974 Pielou (see [22, p. 194]) considered the delay difference equation

$$
N_{t+1}=\frac{\alpha N_{t}}{1+\beta N_{t-k}}, \quad \alpha \in(1, \infty), \beta \in(0, \infty), k \in \mathbb{N}
$$

as the discrete analogue of the delay logistic equation

$$
N^{\prime}(t)=r N(t)\left[1-\frac{N(t-\tau)}{K}\right],
$$

where $r$ and $K$ are the growth rate and the carrying capacity of the population, respectively. Pielou's interest in (3) was in showing that "the tendency to oscillate is a property of the populations themselves and is independent of the extrinsic factors." That is, population sizes oscillate "even though the environment remains constant." According to Pielou, "oscillations can be set up in a population if its growth rate is governed by a density dependent mechanism and if there is a delay in the response of the growth rate to density changes. When this happens the size of the population alternately overshoots and undershoots its equilibrium level."

The blowfly (Lucilia cuprina) studied in 1954 by Nicholson (see [22, p. 194]) is an example of a laboratory population which behaves in the manner described above.

It is noteworthy that a first-order linear difference equation of the form $(1)^{\prime}$ without retarded $(\tau(n) \equiv n)$ argument does not possess oscillatory solutions when $p(n)<1$. A small delay may change this situation, as one can see below, even in the case of equations with constant delays and constant coefficients, and this certainly adds interest in the investigation of the oscillatory character of the above equations (1) and (1)'.

A great part of the existing literature on the oscillation of (1)' concerns the case where the argument $\tau(n)$ is nondecreasing, while only a small number of papers are dealing with the general case of arguments being not necessarily monotone. See, for example, $[4-6,11,12,30]$ and the references cited therein. The consideration of nonmonotone arguments may lead to better approximation of the natural phenomena described by difference equations because quite often there appear natural disturbances (e.g., noise in communication systems) that affect all the parameters of the equation and therefore the "fair" (from a mathematical point of view) monotone arguments are, in fact, nonmonotone almost always. In view of this, for the case of (1)' an interesting question is whether we can state oscillation criteria considering the argument $\tau(n)$ to be not necessarily monotone.

In this paper, we present a survey on the oscillation of solutions to (1) and (1) $)^{\prime}$ in both cases that the argument $\tau(n)$ is monotone or nonmonotone and examples which illustrate the significance of the results.

\section{Oscillation Criteria for (1)}

In 1981, Domshlak [15] studied this problem in the case where $k=1$. Then, in 1989, Erbe and Zhang [21] established the following oscillation criteria for (1).
Theorem 1 (see [21]). Assume that

$$
\beta:=\liminf _{n \rightarrow \infty} p(n)>0, \quad \limsup _{n \rightarrow \infty} p(n)>1-\beta
$$

or

$$
\liminf _{n \rightarrow \infty} p(n)>\frac{k^{k}}{(k+1)^{k+1}}
$$

or

$$
A:=\limsup _{n \rightarrow \infty} \sum_{i=n-k}^{n} p(i)>1 .
$$

Then all solutions of (1) oscillate.

In the same year 1989 Ladas et al. [25] proved the following theorem.

Theorem 2 (see [25]). Assume that

$$
\liminf _{n \rightarrow \infty} \frac{1}{k_{i=n-k}} \sum^{n-1} p(i)>\frac{k^{k}}{(k+1)^{k+1}} .
$$

Then all solutions of (1) oscillate.

Therefore they improved the condition $\left(C_{2}\right)$ by replacing the $p(n)$ of $\left(C_{2}\right)$ by the arithmetic mean of the terms $p(n-$ $k), \ldots, p(n-1)$ in $\left(C_{4}\right)$.

Note that this condition is sharp in the sense that the fraction on the right hand side cannot be improved, since when $p(n)$ is a constant, say $p(n)=p$, then this condition reduces to

$$
p>\frac{k^{k}}{(k+1)^{k+1}},
$$

which is a necessary and sufficient condition for the oscillation of all solutions to (1). Moreover, concerning the constant $k^{k} /(k+1)^{k+1}$ in $\left(C_{2}\right)$ and $\left(C_{4}\right)$ it should be emphasized that, as it is shown in [21], if

$$
\sup p(n)<\frac{k^{k}}{(k+1)^{k+1}},
$$

then (1) has a nonoscillatory solution.

In 1990, Ladas [24] conjectured that (1) has a nonoscillatory solution if

$$
\frac{1}{k} \sum_{i=n-k}^{n-1} p(i) \leq \frac{k^{k}}{(k+1)^{k+1}}
$$

holds eventually. However this conjecture is not correct and a counterexample was given in 1994 by Yu et al. [36]. Moreover, in 1999 Tang and $\mathrm{Yu}$ [33], using a different technique, showed that if

$$
\sum_{i=n-k}^{n} p(i) \leq \frac{1}{e} \text { for all large } n,
$$


then (1) has a nonoscillatory solution. Therefore, as a corollary (see [33] Corollary 2]), Tang and Yu presented an affirmative answer to the so-called "corrected Ladas conjecture"; that is, if

$$
\sum_{i=n-k}^{n} p(i) \leq\left(\frac{k}{k+1}\right)^{k+1} \quad \text { for all large } n
$$

then (1) has a nonoscillatory solution.

Very recently Karpuz [23] studied this problem and derived the following conditions. If

$$
\liminf _{n \rightarrow \infty} \inf _{\lambda \geq 1}\left[\frac{1}{\lambda} \prod_{i=n-k}^{n}[1+\lambda p(i)]\right]>1,
$$

then every solution of (1) oscillates, while if there exists $\lambda_{0} \geq 1$ such that

$$
\frac{1}{\lambda_{0}} \prod_{i=n-k}^{n}\left[1+\lambda_{0} p(i)\right] \leq 1 \quad \text { for all large } n,
$$

then (1) has a nonoscillatory solution.

From the above conditions, using the arithmetic-geometric mean, it follows that if

$$
\sum_{i=n-k}^{n} p(i) \leq\left(\frac{k}{k+1}\right)^{k} \text { for all large } n,
$$

then (1) has a nonoscillatory solution. That is, Karpuz replaced condition $\left(N_{2}\right)$ by $\left(N_{3}\right)$, which is a weaker condition.

It is interesting to establish sufficient conditions for the oscillation of all solutions of (1) when both $\left(C_{3}\right)$ and $\left(C_{4}\right)$ are not satisfied. (For (2) and (2)' this question has been investigated by many authors; see, e.g., [30] and the references cited therein.)

In 1993, Yu et al. [35] and Lalli and Zhang [26], trying to improve $\left(C_{3}\right)$, established the following (false) sufficient oscillation conditions for (1)

$$
\begin{gathered}
0<\alpha:=\liminf _{n \rightarrow \infty} \sum_{i=n-k}^{n-1} p(i) \leq\left(\frac{k}{k+1}\right)^{k+1}, \quad A>1-\frac{\alpha^{2}}{4}, \\
\sum_{i=n-k}^{n} p(i) \geq d>0 \text { for large } n \\
A>1-\frac{d^{4}}{8}\left(1-\frac{d^{3}}{4}+\sqrt{1-\frac{d^{3}}{2}}\right)^{-1}
\end{gathered}
$$

respectively.

Unfortunately, the above conditions $\left(F_{1}\right)$ and $\left(F_{2}\right)$ are not correct. This is due to the fact that they are based on the following (false) discrete version of Koplatadze-Chanturia Lemma. (See $[14,18]$.)

Lemma A (false). Assume that $x(n)$ is an eventually positive solution of (1) and that

$$
\sum_{i=n-k}^{n} p(i) \geq M>0 \quad \text { for large } n
$$

Then

$$
x(n)>\frac{M^{2}}{4} x(n-k) \quad \text { for large } n .
$$

As one can see, the erroneous proof of Lemma A is based on the following (false) statement. (See $[14,18]$.)

Statement $A$ (False). If (9) holds, then for any large $N$, there exists a positive integer $n$ such that $n-k \leq N \leq n$ and

$$
\sum_{i=n-k}^{N} p(i) \geq \frac{M}{2}, \quad \sum_{i=N}^{n} p(i) \geq \frac{M}{2} .
$$

It is obvious that all the oscillation results which have made use of Lemma A or Statement A are incorrect. For details on this problem see the paper by Cheng and Zhang [14].

Here it should be pointed out that the following statement (see $[25,28]$ ) is correct and it should not be confused with Statement A.

Statement 3 (see $[25,28])$. If

$$
\sum_{i=n-k}^{n-1} p(i) \geq M>0 \text { for large } n,
$$

then for any large $n$, there exists a positive integer $n^{*}$ with $n-k \leq n^{*} \leq n$ such that

$$
\sum_{i=n-k}^{n^{*}} p(i) \geq \frac{M}{2}, \quad \sum_{i=n^{*}}^{n} p(i) \geq \frac{M}{2} .
$$

In 1995, Stavroulakis [28], based on this correct Statement 3 , proved the following theorem.

Theorem 4 (see [28]). Assume that

$$
\begin{gathered}
0<\alpha \leq\left(\frac{k}{k+1}\right)^{k+1}, \\
\limsup _{n \rightarrow \infty} p(n)>1-\frac{\alpha^{2}}{4} .
\end{gathered}
$$

Then all solutions of (1) oscillate.

In 1999 Domshlak [18] and in 2000 Cheng and Zhang [14] established the following lemmas, respectively, which may be looked upon as (exact) discrete versions of KoplatadzeChanturia Lemma.

Lemma 5 (see [18]). Assume that $x(n)$ is an eventually positive solution of (1) and that condition (12) holds. Then

$$
x(n)>\frac{M^{2}}{4} x(n-k) \quad \text { for large } n .
$$

Lemma 6 (see [14]). Assume that $x(n)$ is an eventually positive solution of (1) and that condition (12) holds. Then

$$
x(n)>M^{k} x(n-k) \quad \text { for large } n .
$$


Based on these lemmas, the following theorem was established in 2004 by Stavroulakis [29].

Theorem 7 (see [29]). Assume that

$$
0<\alpha \leq\left(\frac{k}{k+1}\right)^{k+1} .
$$

Then either one of the conditions

$$
\limsup _{n \rightarrow \infty} \sum_{i=n-k}^{n-1} p(i)>1-\frac{\alpha^{2}}{4}
$$

or

$$
\limsup _{n \rightarrow \infty} \sum_{i=n-k}^{n-1} p(i)>1-\alpha^{k}
$$

implies that all solutions of (1) oscillate.

Remark 8 (see [29]). From the above theorem it is now clear that

$$
\begin{gathered}
0<\alpha:=\liminf _{n \rightarrow \infty} \sum_{i=n-k}^{n-1} p(i) \leq\left(\frac{k}{k+1}\right)^{k+1}, \\
\limsup _{n \rightarrow \infty} \sum_{i=n-k}^{n-1} p(i)>1-\frac{\alpha^{2}}{4}
\end{gathered}
$$

is the correct oscillation condition by which the (false) condition $\left(F_{1}\right)$ should be replaced.

Remark 9 (see [29]). Observe the following:

(i) When $k=1,2$,

$$
\alpha^{k}>\frac{\alpha^{2}}{4}
$$

(since, from the above-mentioned conditions, it makes sense to investigate the case when $\left.\alpha<(k /(k+1))^{k+1}\right)$ and therefore condition $\left(C_{6}\right)$ implies $\left(C_{7}\right)$.

(ii) When $k=3$,

$$
\alpha^{3}>\frac{\alpha^{2}}{4} \quad \text { when } \alpha>\frac{1}{4}
$$

while

$$
\alpha^{3}<\frac{\alpha^{2}}{4} \quad \text { when } \alpha<\frac{1}{4} .
$$

Therefore, in this case conditions $\left(C_{6}\right)$ and $\left(C_{7}\right)$ are independent.

(iii) When $k \geq 4$,

$$
\alpha^{k}<\frac{\alpha^{2}}{4}
$$

and therefore condition $\left(C_{7}\right)$ implies $\left(C_{6}\right)$. (iv) When $k<12$, condition $\left(C_{6}\right)$ or $\left(C_{7}\right)$ implies $\left(C_{3}\right)$.

(v) When $k \geq 12$, condition $\left(C_{6}\right)$ may hold but condition $\left(C_{3}\right)$ may not hold.

We illustrate these by the following examples.

Example 10 (see [29]). Consider the equation

$$
x(n+1)-x(n)+p(n) x(n-3)=0,
$$

where

$$
\begin{aligned}
& p(2 n)=\frac{1}{10}, \\
& p(2 n+1)=\frac{1}{10}+\frac{64}{95} \sin ^{2} \frac{n \pi}{2}, \\
& n=0,1,2, \ldots
\end{aligned}
$$

Here $k=3$ and it is easy to see that

$$
\begin{gathered}
\alpha=\liminf _{n \rightarrow \infty} \sum_{i=n-3}^{n-1} p(i)=\frac{3}{10}<\left(\frac{3}{4}\right)^{4}, \\
\limsup _{n \rightarrow \infty} \sum_{i=n-3}^{n-1} p(i)=\frac{3}{10}+\frac{64}{95}>1-\alpha^{3} .
\end{gathered}
$$

Thus condition $\left(C_{7}\right)$ is satisfied and therefore all solutions oscillate. Observe, however, that condition $\left(C_{6}\right)$ is not satisfied.

If, on the other hand, in the above equation

$$
\begin{aligned}
p(2 n) & =\frac{8}{100}, \\
p(2 n+1) & =\frac{8}{100}+\frac{746}{1000} \sin ^{2} \frac{n \pi}{2}, \\
n & =0,1,2, \ldots,
\end{aligned}
$$

then it is easy to see that

$$
\alpha=\liminf _{n \rightarrow \infty} \sum_{i=n-3}^{n-1} p(i)=\frac{24}{100}<\left(\frac{3}{4}\right)^{4},
$$

$$
\limsup _{n \rightarrow \infty} \sum_{i=n-3}^{n-1} p(i)=\frac{24}{100}+\frac{746}{1000}>1-\frac{\alpha^{2}}{4} \text {. }
$$

In this case condition $\left(C_{6}\right)$ is satisfied and therefore all solutions oscillate. Observe, however, that condition $\left(C_{7}\right)$ is not satisfied.

Example 11 (see [29]). Consider the equation

$$
x(n+1)-x(n)+p(n) x(n-16)=0,
$$


where

$$
\begin{gathered}
p(17 n)=p(17 n+1)=\cdots=p(17 n+15)=\frac{2}{100}, \\
p(17 n+16)=\frac{2}{100}+\frac{655}{1000}, \\
n=0,1,2, \ldots .
\end{gathered}
$$

Here $k=16$ and it is easy to see that

$$
\begin{gathered}
\alpha=\liminf _{n \rightarrow \infty} \sum_{i=n-16}^{n-1} p(i)=\frac{32}{100}<\left(\frac{16}{17}\right)^{17}, \\
\underset{n \rightarrow \infty}{\limsup } \sum_{i=n-16}^{n-1} p(i)=\frac{32}{100}+\frac{655}{1000}=0.975>1-\frac{\alpha^{2}}{4} .
\end{gathered}
$$

We see that condition $\left(C_{6}\right)$ is satisfied and therefore all solutions oscillate. Observe, however, that

$$
A=\limsup _{n \rightarrow \infty} \sum_{i=n-16}^{n} p(i)=\frac{34}{100}+\frac{655}{1000}=0.995<1 ;
$$

that is, condition $\left(C_{3}\right)$ is not satisfied.

In 1995, Chen and Yu [13], following the above-mentioned direction, derived a condition which, formulated in terms of $\alpha$ and $A$, says that all solutions of (1) oscillate if $0<\alpha \leq$ $k^{k+1} /(k+1)^{k+1}$ and

$$
A>1-\frac{1-\alpha-\sqrt{1-2 \alpha-\alpha^{2}}}{2} .
$$

In 1998, Domshlak [17] studied the oscillation of all solutions and the existence of nonoscillatory solution of (1) with $r$-periodic positive coefficients $p(n), p(n+r)=p(n)$. It is very important that, in the following cases where $\{r=$ $k\},\{r=k+1\},\{r=2\},\{k=1, r=3\}$, and $\{k=1, r=4\}$, the results obtained are stated in terms of necessary and sufficient conditions and it is very easy to check them.

In 2000, Tang and $\mathrm{Yu}$ [34] improved condition $\left(C_{8}\right)$ to the condition

$$
A>\lambda_{2}^{k}\left(1-k \ln \lambda_{2}\right)-\frac{1-\alpha-\sqrt{1-\alpha-\alpha^{2}}}{2},
$$

where $\lambda_{2}$ is the greater root of the algebraic equation

$$
k \lambda^{k}(1-\lambda)=\alpha
$$

In 2001, Shen and Stavroulakis [27], using new techniques, improved the previous results as follows.

Theorem 12 (see [27]). Assume that $0 \leq \alpha \leq k^{k+1} /(k+1)^{k+1}$ and that there exists an integer $l \geq 1$ such that

$$
\begin{array}{r}
\limsup _{n \rightarrow \infty}\left\{\sum_{i=1}^{k} p(n-i)+[\bar{d}(\alpha)]^{-k} \prod_{i=1}^{k} \sum_{j=1}^{k} p(n-i+j)\right. \\
\left.+\sum_{m=0}^{l-1}\left[d\left(\frac{\alpha}{k}\right)\right]^{-(m+1) k} \sum_{i=1}^{k} \prod_{j=0}^{m+1} p(n-k j+i)\right\}>1,
\end{array}
$$

where $\bar{d}(\alpha)$ and $d(\alpha / k)$ are the greater real roots of the equations

$$
\begin{aligned}
& d^{k+1}-d^{k}+\alpha^{k}=0 \\
& d^{k+1}-d^{k}+\frac{\alpha}{k}=0
\end{aligned}
$$

respectively. Then all solutions of (1) oscillate.

Notice that when $k=1, d(\alpha)=\bar{d}(\alpha)=(1+\sqrt{1-4 \alpha}) / 2$ (see [27]), and so condition $\left(C_{10}\right)$ reduces to

$$
\begin{aligned}
\limsup _{n \rightarrow \infty} & \{C p(n)+p(n-1) \\
& \left.+\sum_{m=0}^{l-1} C^{m+1} \prod_{j=0}^{m+1} p(n-j-1)\right\}>1,
\end{aligned}
$$

where $C=2 /(1+\sqrt{1-4 \alpha}), \alpha=\liminf _{n \rightarrow \infty} p_{n}$. Therefore, from Theorem 12, we have the following corollary.

Corollary 13 (see [27]). Assume that $0 \leq \alpha \leq 1 / 4$ and that $\left(C_{11}\right)$ holds. Then all solutions of the equation

$$
x(n+1)-x(n)+p(n) x(n-1)=0
$$

oscillate.

A condition derived from $\left(C_{11}\right)$ and which can be easily verified is given in the next corollary.

Corollary 14 (see [27]). Assume that $0 \leq \alpha \leq 1 / 4$ and that

$$
\limsup _{n \rightarrow \infty} p(n)>\left(\frac{1+\sqrt{1-4 \alpha}}{2}\right)^{2} \text {. }
$$

Then all solutions of (34) oscillate.

Remark 15 (see [27]). Observe that when $\alpha=1 / 4$, condition $\left(C_{12}\right)$ reduces to

$$
\limsup _{n \rightarrow \infty} p(n)>\frac{1}{4}
$$

which cannot be improved in the sense that the lower bound $1 / 4$ cannot be replaced by a smaller number. Indeed, by condition $\left(N_{1}\right)$ (Theorem 2.3 in [21]), we see that (34) has a nonoscillatory solution if

$$
\sup p(n)<\frac{1}{4}
$$

Note, however, that even in the critical state where

$$
\lim _{n \rightarrow \infty} p(n)=\frac{1}{4}
$$

(34) can be either oscillatory or nonoscillatory. For example, if $p(n)=1 / 4+c / n^{2}$, then (34) will be oscillatory in case $c>1 / 4$ and nonoscillatory in case $c<1 / 4$ (the Kneser-like theorem, [16]). 
Example 16 (see [27]). Consider the equation

$$
x(n-1)-x(n)+\left(\frac{1}{4}+a \sin ^{4} \frac{n \pi}{8}\right) x(n-1)=0,
$$

where $a>0$ is a constant. It is easy to see that

$$
\begin{aligned}
& \liminf _{n \rightarrow \infty} p(n)=\liminf _{n \rightarrow \infty}\left(\frac{1}{4}+a \sin ^{4} \frac{n \pi}{8}\right)=\frac{1}{4} \\
& \limsup _{n \rightarrow \infty} p(n)=\limsup _{n \rightarrow \infty}\left(\frac{1}{4}+a \sin ^{4} \frac{n \pi}{8}\right)=\frac{1}{4}+a .
\end{aligned}
$$

Therefore, by Corollary 14, all solutions oscillate. However, none of the conditions $\left(C_{1}\right)-\left(C_{9}\right)$ is satisfied.

The following corollary concerns the case when $k>1$.

Corollary 17 (see [27]). Assume that $0 \leq \alpha \leq k^{k+1} /(k+1)^{k+1}$ and that

$$
\begin{aligned}
\limsup _{n \rightarrow \infty} \sum_{i=n-k}^{n-1} p(i)>1 & -[\bar{d}(\alpha)]^{-k} \alpha^{k} \\
& -\frac{k[d(\alpha / k)]^{-k} \beta^{2}}{1-[d(\alpha / k)]^{-k} \beta},
\end{aligned}
$$

where $\bar{d}(\alpha), d(\alpha / k)$ are as in Theorem 12. Then all solutions of (1) oscillate.

Following this historical (and chronological) review we also mention that in the (critical) case where

$$
\begin{aligned}
\frac{1}{k} \sum_{i=n-k}^{n-1} p(i) & \geq \frac{k^{k}}{(k+1)^{k+1},} \\
\lim _{n \rightarrow \infty} \frac{1}{k} \sum_{i=n-k}^{n-1} p(i) & =\frac{k^{k}}{(k+1)^{k+1}},
\end{aligned}
$$

the oscillation of (1) has been studied in 1994 by Domshlak [16] and in 1998 by Tang [31] (see also Tang and Yu [32]). In a case when $p(n)$ is asymptotically close to one of the periodic critical states, unimprovable results about oscillation properties of the equation

$$
x(n+1)-x(n)+p(n) x(n-1)=0
$$

were obtained by Domshlak in 1999 [19] and in 2000 [20].

\section{Oscillation Criteria for $(1)^{\prime}$}

In this section we study the delay difference equation with variable argument $(1)^{\prime}$ where $\Delta x(n)=x(n+1)-x(n), p(n)$ is a sequence of nonnegative real numbers and $\tau(n)$ is a sequence of integers such that $\tau(n) \leq n-1$ for all $n \geq 0$ and $\lim _{n \rightarrow \infty} \tau(n)=\infty$.

In 2008, Chatzarakis et al. [7] investigated for the first time the oscillatory behavior of equation (1) ${ }^{\prime}$ in the case of a general nonmonotone delay argument $\tau(n)$ and derived the following theorem.
Theorem 18 (see [7]). Assume that

$$
\sigma(n):=\max _{0 \leq s \leq n} \tau(s), \quad n \geq 0 .
$$

If

$$
\limsup _{n \rightarrow \infty} \sum_{j=\sigma(n)}^{n} p(j)>1,
$$

then all solutions of equation (1)' oscillate.

Remark 19 (see [7]). Clearly, the sequence of integers $\sigma(n)$ is nondecreasing and $\tau(n) \leq \sigma(n)$ for all $n \geq 0$.

Also in the same year Chatzarakis et al. [8] derived the following theorem.

Theorem 20 (see [8]). Assume that

$$
\begin{gathered}
\limsup _{n \rightarrow \infty} \sum_{i=\tau(n)}^{n-1} p(i)<+\infty, \\
\boldsymbol{\alpha}:=\liminf _{n \rightarrow \infty} \sum_{i=\tau(n)}^{n-1} p(i)>\frac{1}{e} .
\end{gathered}
$$

Then all solutions of $(1)^{\prime}$ oscillate.

Remark 21 (see [8]). Note that condition (43) is not a limitation since, by $\left(C_{1}^{\prime}\right)$, if $\tau$ is a nondecreasing function, all solutions of $(1)^{\prime}$ oscillate.

Remark 22 (see [8]). Condition $\left(C_{2}^{\prime}\right)$ is optimal for $(1)^{\prime}$ under the assumption that $\lim _{n \rightarrow+\infty}(n-\tau(n))=\infty$, since in this case the set of natural numbers increases infinitely in the interval $[\tau(n), n-1]$ for $n \rightarrow \infty$.

In [8] an example is also presented to show that the condition $\left(C_{2}^{\prime}\right)$ is optimal, in the sense that it cannot be replaced by the nonstrong inequality.

As it has been mentioned above, it is an interesting problem to find new sufficient conditions for the oscillation of all solutions of the delay difference equation $(1)^{\prime}$, in the case where neither $\left(C_{1}^{\prime}\right)$ nor $\left(C_{2}^{\prime}\right)$ is satisfied.

In 2008 Chatzarakis et al. [7] and in 2008 and 2009 Chatzarakis et al. $[9,10]$ derived the following conditions.

Theorem 23 (see $[7,9,10]$ ). (I) Assume that $0<\boldsymbol{\alpha} \leq 1 / e$. Then either one of the conditions:

$$
\begin{aligned}
& \limsup _{n \rightarrow \infty} \sum_{j=\sigma(n)}^{n} p(j)>1-(1-\sqrt{1-\boldsymbol{\alpha}})^{2}, \\
& \limsup _{n \rightarrow \infty} \sum_{j=\sigma(n)}^{n} p(j)>1-\frac{1}{2}(1-\boldsymbol{\alpha}-\sqrt{1-2 \boldsymbol{\alpha}}),
\end{aligned}
$$




$$
\limsup _{n \rightarrow \infty} \sum_{j=\sigma(n)}^{n} p(j)>1-\frac{1}{2}\left(1-\boldsymbol{\alpha}-\sqrt{1-2 \boldsymbol{\alpha}-\boldsymbol{\alpha}^{2}}\right), \quad\left(C_{5}^{\prime}\right)
$$

implies that all solutions of (1)' oscillate.

(II) If $0<\boldsymbol{\alpha} \leq 1 / e$ and, in addition, $p(n) \geq 1-\sqrt{1-\boldsymbol{\alpha}}$ for all large $n$ and

$$
\limsup _{n \rightarrow \infty} \sum_{j=\sigma(n)}^{n} p(j)>1-\boldsymbol{\alpha} \frac{1-\sqrt{1-\boldsymbol{\alpha}}}{\sqrt{1-\boldsymbol{\alpha}}}
$$

or if $0<\boldsymbol{\alpha} \leq 6-4 \sqrt{2}$ and, in addition, $p(n) \geq \boldsymbol{\alpha} / 2$ for all large $n$ and

$$
\limsup _{n \rightarrow \infty} \sum_{j=\sigma(n)}^{n} p(j)>1-\frac{1}{4}\left(2-3 \boldsymbol{\alpha}-\sqrt{4-12 \boldsymbol{\alpha}+\boldsymbol{\alpha}^{2}}\right),
$$

then all solutions of $(1)^{\prime}$ are oscillatory.

Remark 24. Observe the following:

(i) When $0<\boldsymbol{\alpha} \leq 1 / e$, it is easy to verify that

$$
\begin{gathered}
\frac{1-\boldsymbol{\alpha}-\sqrt{1-2 \boldsymbol{\alpha}-\boldsymbol{\alpha}^{2}}}{2}>\boldsymbol{\alpha} \frac{1-\sqrt{1-\boldsymbol{\alpha}}}{\sqrt{1-\boldsymbol{\alpha}}} \\
>\frac{1-\boldsymbol{\alpha}-\sqrt{1-2 \alpha}}{2}>(1-\sqrt{1-\boldsymbol{\alpha}})^{2}
\end{gathered}
$$

and therefore condition $\left(C_{5}^{\prime}\right)$ is weaker than conditions (46), (45), and (44).

(ii) When $0<\boldsymbol{\alpha} \leq 6-4 \sqrt{2}$, it is easy to show that

$$
\begin{aligned}
\frac{1}{4}\left(2-3 \boldsymbol{\alpha}-\sqrt{4-12 \boldsymbol{\alpha}+\boldsymbol{\alpha}^{2}}\right) \\
>\frac{1}{2}\left(1-\boldsymbol{\alpha}-\sqrt{1-2 \boldsymbol{\alpha}-\boldsymbol{\alpha}^{2}}\right)
\end{aligned}
$$

and therefore, in this case, inequality (47) improves inequality $\left(C_{5}^{\prime}\right)$ and, especially, when $\boldsymbol{\alpha}=6-4 \sqrt{2} \simeq 0.3431457$, the lower bound in $\left(C_{5}^{\prime}\right)$ is 0.8929094 while that in $(47)$ is 0.7573593 .

In 2011, Braverman and Karpuz [6] studied (1)' also in the case of nonmonotone delays. More precisely the following were derived in [6].

Theorem 25 (see [6]). There is no constant $\Lambda>0$ such that the inequalities

$$
\begin{array}{r}
\limsup _{n \rightarrow \infty}(n-\tau(n)) p(n)>\Lambda, \\
\limsup _{n \rightarrow \infty} \sum_{i=\tau(n)}^{n-1} p(i)>\Lambda
\end{array}
$$

imply oscillation of $(1)^{\prime}$.

Remark 26 (see [6]). Obviously, there is no constant $\Lambda>0$ such that

$$
\limsup _{n \rightarrow \infty} \sum_{i=\tau(n)}^{n} p(i)>\Lambda
$$

implies oscillation of $(1)^{\prime}$.

Remark 27. At this point it should be emphasized that conditions $\left(C_{1}^{\prime}\right)$ and $\left(C_{2}^{\prime}\right)$ imply that all solutions of $(1)^{\prime}$ oscillate without the assumption that $\tau(n)$ is monotone. Note that in $\left(C_{1}^{\prime}\right)$ instead of $\tau(n)$ the sequence $\sigma(n)$, defined by (42), is considered, which is nondecreasing and $\tau(n) \leq \sigma(n)$ for all $n \geq 0$.

Theorem 28 (see [6]). If

$$
\limsup _{n \rightarrow \infty} \sum_{j=\sigma(n)}^{n} p(j) \prod_{i=\tau(j)}^{\sigma(n)-1} \frac{1}{1-p(i)}>1,
$$

then every solution of $(1)^{\prime}$ oscillates.

Using the upper bound of the ratio $x(\tau(n)) / x(n)$ for possible nonoscillatory solutions $x(n)$ of $(1)^{\prime}$, presented in [7-10], the above result was essentially improved in 2014 by Stavroulakis [30].

Theorem 29 (see [30]). Assume that

$$
\boldsymbol{\alpha}=\liminf _{n \rightarrow \infty} \sum_{i=\tau(n)}^{n-1} p(i)
$$

$$
\limsup _{n \rightarrow \infty} \sum_{j=\sigma(n)}^{n} p(j) \prod_{i=\tau(j)}^{\sigma(n)-1} \frac{1}{1-p(i)}>1-c(\boldsymbol{\alpha})
$$

where

$$
c(\boldsymbol{\alpha})= \begin{cases}\frac{1}{2}\left(1-\boldsymbol{\alpha}-\sqrt{1-2 \boldsymbol{\alpha}-\boldsymbol{\alpha}^{2}}\right) & \text { if } 0<\boldsymbol{\alpha} \leq 1 / e, \\ \frac{1}{4}\left(2-3 \boldsymbol{\alpha}-\sqrt{4-12 \boldsymbol{\alpha}+\boldsymbol{\alpha}^{2}}\right) & \text { if } 0<\boldsymbol{\alpha} \leq 6-4 \sqrt{2}, p(n) \geq \frac{\boldsymbol{\alpha}}{2} .\end{cases}
$$

Then all solutions of $(1)^{\prime}$ oscillate. 
Remark 30. Observe that, as $\boldsymbol{\alpha} \rightarrow 0$, condition (54) reduces to (52). However the improvement is clear as $\boldsymbol{\alpha} \rightarrow 1 / e$. Actually, when $\boldsymbol{\alpha}=1 / e$, the value of the lower bound in (54) is equal to $\approx 0.863457014$, while when $p(n) \geq \boldsymbol{\alpha} / 2$ and $\boldsymbol{\alpha}=6-4 \sqrt{2} \simeq$ 0.3431457 , the lower bound in (54) is 0.7573593 . That is, in all cases condition (54) of Theorem 29 essentially improves condition (52) of Theorem 28.

Example 31 (see [30] cf. [6]). Consider the equation

$$
\Delta x(n)+p(n) x(\tau(n))=0, \quad n=0,1,2, \ldots,
$$

where

$$
\begin{gathered}
p(n)= \begin{cases}10^{-4}, & n \text { is even } \\
\frac{1}{e}, & n \text { is odd }\end{cases} \\
\tau(n)= \begin{cases}n-1, & n \text { is even } \\
n-2, & n \text { is odd. }\end{cases}
\end{gathered}
$$

Observe that for this equation, $\sigma(n)=\tau(n)$ and it is easy to see that

$$
\begin{array}{r}
\sum_{i=\tau(n)}^{n-1} p(i)= \begin{cases}\frac{1}{e}, & n \text { is even } \\
\frac{1}{e}+10^{-4}, & n \text { is odd },\end{cases} \\
\sum_{i=\tau(n)}^{n} p(i)= \begin{cases}\frac{1}{e}+10^{-4}, & n \text { is even } \\
\frac{2}{e}+10^{-4}, & n \text { is odd }\end{cases} \\
\sum_{j=\sigma(n)}^{n} p(j) \prod_{i=\tau(j)}^{\sigma(n)-1} \frac{1}{1-p(i)} \simeq \begin{cases}0.58, & n \text { is even } \\
0.95, & n \text { is odd }\end{cases}
\end{array}
$$

and so

$$
\begin{aligned}
& \limsup _{n \rightarrow \infty} \sum_{i=\tau(n)}^{n} p(i)=\frac{2}{e}+10^{-4} \simeq 0.7358, \\
& \limsup _{n \rightarrow \infty} \sum_{i=\tau(n)}^{n-1} p(i)=\frac{1}{e}+10^{-4} \simeq 0.3679, \\
& \liminf _{n \rightarrow \infty} \sum_{i=\tau(n)}^{n-1} p(i)=\frac{1}{e}, \\
& \limsup _{n \rightarrow \infty} \sum_{j=\sigma(n)}^{n} p(j) \prod_{i=\tau(j)}^{\sigma(n)-1} \frac{1}{1-p(i)} \simeq 0.95 .
\end{aligned}
$$

Thus,

$$
\begin{array}{r}
0.7358<1 \\
\boldsymbol{\alpha}=\frac{1}{e},
\end{array}
$$

$$
\begin{aligned}
0.7358 & <1-(1-\sqrt{1-\boldsymbol{\alpha}})^{2} \simeq 0.9579 \\
0.7358 & <1-\frac{1}{2}(1-\boldsymbol{\alpha}-\sqrt{1-2 \boldsymbol{\alpha}}) \simeq 0.9409 \\
0.7358 & <1-\frac{1}{2}\left(1-\boldsymbol{\alpha}-\sqrt{1-2 \boldsymbol{\alpha}-\boldsymbol{\alpha}^{2}}\right) \simeq 0.8634 \\
0.95 & <1
\end{aligned}
$$

and therefore none of the known oscillation conditions $\left(C_{1}^{\prime}\right)$, $\left(C_{2}^{\prime}\right),(44),(45),\left(C_{5}^{\prime}\right)$, and (52) is satisfied. However,

$$
\begin{aligned}
& \limsup _{n \rightarrow \infty} \sum_{j=\sigma(n)}^{n} p(j) \prod_{i=\tau(j)}^{\sigma(n)-1} \frac{1}{1-p(i)} \simeq 0.95 \\
& >1-\frac{1}{2}\left(1-\boldsymbol{\alpha}-\sqrt{1-2 \boldsymbol{\alpha}-\boldsymbol{\alpha}^{2}}\right) \simeq 0.8634
\end{aligned}
$$

that is, the conditions of Theorem 29 are satisfied and therefore all solutions to (56) oscillate.

In 2015, Braverman et al. [5] established the following iterative oscillation conditions. If for some $r \in \mathbb{N}$

$$
\underset{n \rightarrow \infty}{\limsup } \sum_{j=\sigma(n)}^{n} p(j) a_{r}^{-1}(\sigma(n), \tau(j))>1,
$$

or

$$
\begin{gathered}
\limsup _{n \rightarrow \infty} \sum_{j=\sigma(n)}^{n} p(j) a_{r}^{-1}(\sigma(n), \tau(j)) \\
>1-\frac{1-\boldsymbol{\alpha}-\sqrt{1-2 \boldsymbol{\alpha}-\boldsymbol{\alpha}^{2}}}{2},
\end{gathered}
$$

where

$$
\begin{aligned}
a_{1}(n, k) & =\prod_{i=k}^{n-1}[1-p(i)], a_{r+1}(n, k) \\
& =\prod_{i=k}^{n-1}\left[1-p(i) a_{r}^{-1}(i, \tau(i))\right]
\end{aligned}
$$

and $\boldsymbol{\alpha}=\liminf _{n \rightarrow \infty} \sum_{j=\tau(n)}^{n-1} p(j)$, then all solutions of $(1)^{\prime}$ oscillate.

In 2017 Asteris and Chatzarakis [4] and Chatzarakis and Shaikhet [12] proved that if for some $\ell \in \mathbb{N}$

$$
\limsup _{n \rightarrow \infty} \sum_{i=\sigma(n)}^{n} p(i) \prod_{j=\tau(i)}^{\sigma(n)-1} \frac{1}{1-p_{\ell}(j)}>1,
$$

or

$$
\begin{gathered}
\limsup _{n \rightarrow \infty} \sum_{i=\sigma(n)}^{n} p(i) \prod_{j=\tau(i)}^{\sigma(n)-1} \frac{1}{1-p_{\ell}(j)} \\
>1-\frac{1-\boldsymbol{\alpha}-\sqrt{1-2 \boldsymbol{\alpha}-\boldsymbol{\alpha}^{2}}}{2}
\end{gathered}
$$


where

$$
p_{\ell}(n)=p(n)\left[1+\sum_{i=\tau(n)}^{n-1} p(i) \prod_{j=\tau(i)}^{\sigma(n)-1} \frac{1}{1-p_{\ell-1}(j)}\right]
$$

with $p_{0}(n)=p(n)$ and $\boldsymbol{\alpha}=\liminf _{n \rightarrow \infty} \sum_{j=\tau(n)}^{n-1} p(j)$, then all solutions of (1)' oscillate.

Very recently Chatzarakis et al. [11] established the following conditions which essentially improve all the related conditions in the literature.

Theorem 32 (see [11]). (i) If there exists an $\ell \geq 1$ such that $P_{\ell}(n) \geq 1$ for sufficiently large $n$, then all solutions of $(1)^{\prime}$ are oscillatory.

(ii) If for some $\ell \in \mathbb{N}$ we have $P_{\ell}(n)<1$, for sufficiently large $n$, and

$$
\limsup _{n \rightarrow \infty} \sum_{i=\sigma(n)}^{n} p(i) \prod_{j=\tau(i)}^{\sigma(n)-1} \frac{1}{1-P_{\ell}(j)}>1,
$$

where

$$
\begin{aligned}
& P_{\ell}(n):=p(n)\left[1+\sum_{i=\tau(n)}^{n-1} p(i)\right. \\
& \left.\quad \cdot \exp \left(\sum_{j=\tau(i)}^{n-1} p(j) \prod_{m=\tau(j)}^{j-1} \frac{1}{1-P_{\ell-1}(m)}\right)\right],
\end{aligned}
$$

then all solutions of $(1)^{\prime}$ are oscillatory.

Theorem 33 (see [11]). Assume that $0<\boldsymbol{\alpha} \leq 1 /$ e and for some $\ell \in \mathbb{N}$

$$
\begin{gathered}
\limsup _{n \rightarrow \infty} \sum_{i=\sigma(n)}^{n} p(i) \prod_{j=\tau(i)}^{\sigma(n)-1} \frac{1}{1-P_{\ell}(j)} \\
>1-\frac{1-\boldsymbol{\alpha}-\sqrt{1-2 \boldsymbol{\alpha}-\boldsymbol{\alpha}^{2}}}{2}
\end{gathered}
$$

or

$$
\begin{gathered}
\limsup _{n \rightarrow \infty} \sum_{i=\sigma(n)}^{n} p(i) \prod_{j=\tau(i)}^{n} \frac{1}{1-P_{\ell}(j)} \\
>\frac{2}{1-\boldsymbol{\alpha}-\sqrt{1-2 \boldsymbol{\alpha}-\boldsymbol{\alpha}^{2}}},
\end{gathered}
$$

where $P_{\ell}(n)$ is defined by (69). Then all solutions of $(1)^{\prime}$ are oscillatory.

The following example illustrates that the conditions of Theorems 32 and 33 essentially improve known results in the literature, yet they indicate a type of independence among some of them. The calculations were made by the use of MATLAB software.
Example 34 (see [11]). Consider the retarded difference equation

$$
\Delta x(n)+p x(\tau(n))=0, \quad n \in \mathbb{N}_{0},
$$

with $0<p \leq 1 / e$, and (see Figure 1(a))

$$
\tau(n)=\left\{\begin{array}{ll}
n-1, & \text { if } n=3 \mu \\
n-2, & \text { if } n=3 \mu+1 \\
n-4, & \text { if } n=3 \mu+2,
\end{array} \quad \mu \in \mathbb{N}_{0} .\right.
$$

Clearly, $\tau$ is nonmonotone. For the function $\sigma$ defined by (42), we have (see, also, Figure 1(b)) that

$$
\sigma(n)=\max _{0 \leq s \leq n} \tau(s)= \begin{cases}n-1, & \text { if } n=3 \mu \\ n-2, & \text { if } n=3 \mu+1 \\ n-3, & \text { if } n=3 \mu+2,\end{cases}
$$

$$
\mu \in \mathbb{N}_{0} .
$$

The left-hand side in (62) attains its maximum at $n=3 \mu+2$, $\mu \in \mathbb{N}_{0}$, for every $r \in \mathbb{N}$. Specifically,

$$
\begin{aligned}
\sum_{j=3 \mu-1}^{3 \mu+2} p(j) a_{2}^{-1}(3 \mu-1, \tau(j)) \\
=p \frac{1}{a_{2}(3 \mu-1, \tau(3 \mu-1))}+p \frac{1}{a_{2}(3 \mu-1, \tau(3 \mu))} \\
\quad+p \frac{1}{a_{2}(3 \mu-1, \tau(3 \mu+1))} \\
\quad+p \frac{1}{a_{2}(3 \mu-1, \tau(3 \mu+2))} \\
=p \frac{1}{a_{2}(3 \mu-1,3 \mu-5)}+p \frac{1}{a_{2}(3 \mu-1,3 \mu-1)} \\
\quad+p \frac{1}{a_{2}(3 \mu-1,3 \mu-1)}+p \frac{1}{a_{2}(3 \mu-1,3 \mu-2)} \\
=p \frac{1}{\prod_{i=3 \mu-5}^{3 \mu-2}\left[1-p a_{1}^{-1}(i, \tau(i))\right]}+p \cdot 1+p \cdot 1 \\
\quad+p \frac{1}{\prod_{i=3 \mu-2}^{3 \mu-2}\left[1-p a_{1}^{-1}(i, \tau(i))\right]}
\end{aligned}
$$




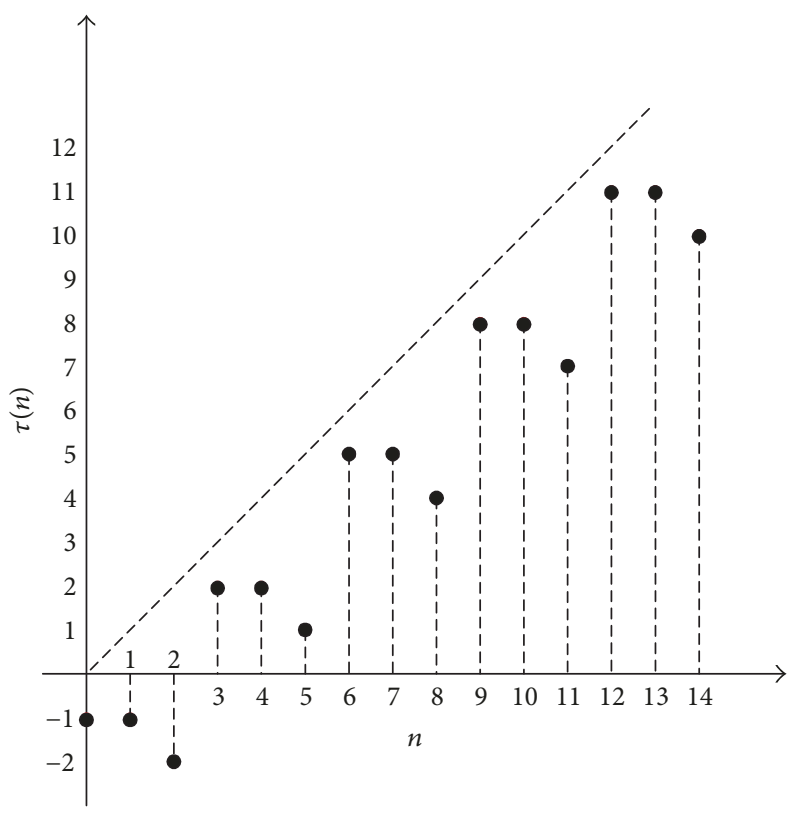

(a)

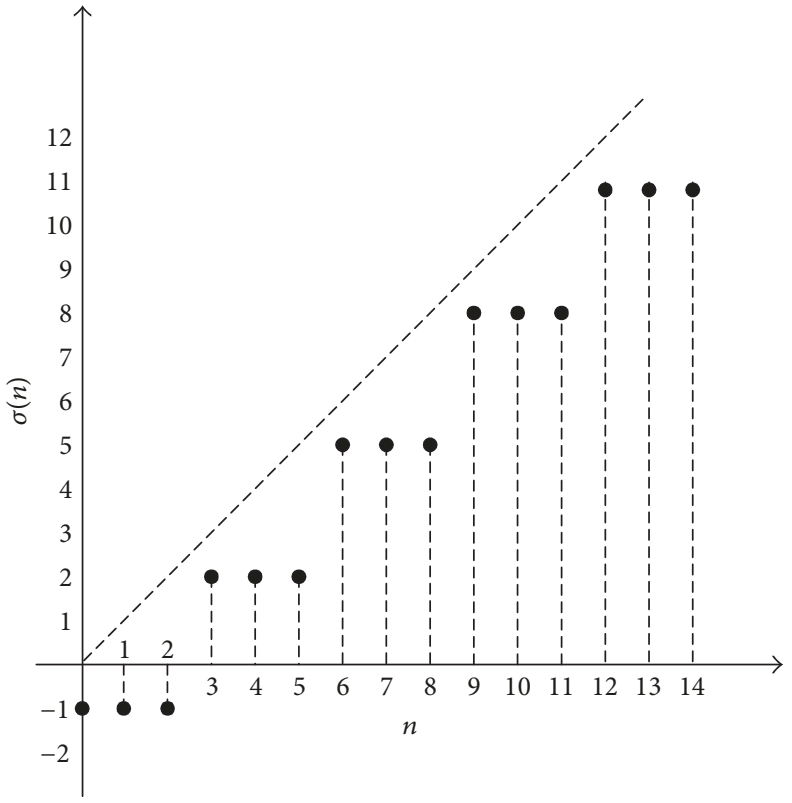

(b)

FIGURE 1: The graphs of $\tau(n)$ and $\sigma(n)$.

$$
\sum_{j=3 \mu-1}^{3 \mu+2} p(j) a_{2}^{-1}(3 \mu-1, \tau(j))=2 p+\frac{p(1-p)^{9}+p(1-p)^{2}\left[(1-p)^{2}-p\right]\left[(1-p)^{4}-p\right](1-2 p)}{\left[(1-p)^{2}-p\right]^{2}\left[(1-p)^{4}-p\right](1-2 p)}
$$

The computation immediately implies that if $p \in[0.145$, $0.1564]$, then

$$
\limsup _{n \rightarrow \infty} \sum_{j=\sigma(n)}^{n} p(j) a_{2}^{-1}(\sigma(n), \tau(j))<1 .
$$

For example, for $p=29 / 200=0.145$, we obtain

$$
\begin{aligned}
& \boldsymbol{\alpha}=\liminf _{n \rightarrow \infty} \sum_{j=\tau(n)}^{n-1} p(j)=\liminf _{\mu \rightarrow \infty} \sum_{j=3 \mu-1}^{3 \mu-1} p(j)=\frac{29}{200} \\
&=0.145<\frac{1}{e} \\
& \limsup _{n \rightarrow \infty} \sum_{j=\sigma(n)}^{n} p(j) a_{2}^{-1}(\sigma(n), \tau(j)) \simeq 0.8437652 \\
& \quad<1, \\
& 0.8437652<1-\frac{1-\boldsymbol{\alpha}-\sqrt{1-2 \boldsymbol{\alpha}-\boldsymbol{\alpha}^{2}}}{2} \simeq 0.9875 .
\end{aligned}
$$

That is, conditions (62) and (63) are not satisfied for $r=$ 2.

Similarly, if $p \in[0.145,0.1564]$, then

$$
\limsup _{n \rightarrow \infty} \sum_{i=\sigma(n)}^{n} p(i) \prod_{j=\tau(i)}^{\sigma(n)-1} \frac{1}{1-p_{1}(j)}<1 .
$$

For example, for $p=29 / 200=0.145$ and by using an algorithm on MATLAB software, we obtain

$$
\begin{aligned}
& \Phi_{1}(n)=\sum_{i=\sigma(n)}^{n} p(i) \prod_{j=\tau(i)}^{\sigma(n)-1} \frac{1}{1-p_{1}(j)}=\sum_{i=\sigma(n)}^{n} p(i) \\
& \cdot \prod_{j=\tau(i)}^{\sigma(n)-1} \frac{1}{1-p(j)\left[1+\sum_{k=\tau(j)}^{j-1} p(k) \prod_{m=\tau(k)}^{\sigma(j)-1}(1 /(1-p(m)))\right]}
\end{aligned}
$$

and therefore

$$
\Phi_{1}(3 \mu+2)=\sum_{i=3 \mu-1}^{3 \mu+2} \frac{29}{200} \prod_{j=\tau(i)}^{3 \mu-2} \frac{1}{1-(29 / 200)\left[1+\sum_{k=\tau(j)}^{j-1}(29 / 200) \prod_{m=\tau(k)}^{\sigma(j)-1}(1 /(1-29 / 200))\right]} \simeq 0.8529 .
$$


Thus

$$
\begin{aligned}
\limsup _{n \rightarrow \infty} & \Phi_{1}(n) \simeq 0.8529<1 \\
0.8529 & <1-\frac{1-\boldsymbol{\alpha}-\sqrt{1-2 \boldsymbol{\alpha}-\boldsymbol{\alpha}^{2}}}{2} \simeq 0.9875 .
\end{aligned}
$$

That is, conditions (65) and (66) are not satisfied for $\ell=1$.
On the contrary, for every $p \in[0.145,0.1564]$, we have

$$
\limsup _{n \rightarrow \infty} \sum_{i=\sigma(n)}^{n} p(i) \prod_{j=\tau(i)}^{\sigma(n)-1} \frac{1}{1-P_{1}(j)}>1
$$

For example, for $p=29 / 200=0.145$ and by using an algorithm on MATLAB software, we obtain

$$
\begin{aligned}
F_{1}(n) & =\sum_{i=\sigma(n)}^{n} p(i) \prod_{j=\tau(i)}^{\sigma(n)-1} \frac{1}{1-P_{1}(j)} \\
& =\sum_{i=\sigma(n)}^{n} p(i) \times \prod_{j=\tau(i)}^{\sigma(n)-1} \frac{1}{1-p(j)\left[1+\sum_{k=\tau(j)}^{j-1} p(k) \exp \left(\sum_{w=\tau(k)}^{j-1} p(w) \prod_{m=\tau(w)}^{w-1}(1 /(1-p(m)))\right)\right]}
\end{aligned}
$$

and therefore

$$
\begin{aligned}
F_{1}(3 \mu+2) & =\sum_{i=3 \mu-1}^{3 \mu+2} \frac{29}{200} \times \prod_{j=\tau(i)}^{3 \mu-2} \frac{1}{1-(29 / 200)\left[1+\sum_{k=\tau(j)}^{j-1}(29 / 200) \exp \left(\sum_{w=\tau(k)}^{j-1}(29 / 200) \prod_{m=\tau(w)}^{w-1}(1 /(1-29 / 200))\right)\right]} \\
& \simeq 1.0248 .
\end{aligned}
$$

Thus

$$
\begin{aligned}
\limsup _{n \rightarrow \infty} F_{1}(n) & =\limsup _{n \rightarrow \infty} \sum_{i=h(n)}^{n} p(i) \prod_{j=\tau(i)}^{\sigma(n)-1} \frac{1}{1-P_{1}(j)} \\
& \simeq 1.0248>1 ;
\end{aligned}
$$

that is, condition (68) of Theorem 32 is satisfied for $\ell=1$. Therefore, all solutions of (72) are oscillatory.

Observe that, for $p=29 / 200=0.145$, we obtain

$$
\begin{aligned}
& \limsup _{n \rightarrow \infty} \sum_{j=\sigma(n)}^{n} p(j)=\limsup _{\mu \rightarrow \infty} \sum_{j=3 \mu-1}^{3 \mu+2} p(j)=4 \cdot \frac{29}{200} \\
& =0.58<1, \\
& \boldsymbol{\alpha}=0.145<\frac{1}{e}, \\
& 0.58<1-\frac{1-\boldsymbol{\alpha}-\sqrt{1-2 \boldsymbol{\alpha}-\boldsymbol{\alpha}^{2}}}{2} \simeq 0.9875, \\
& \limsup _{n \rightarrow \infty} \sum_{j=\sigma(n)}^{n} p(j) \prod_{i=\tau(j)}^{\sigma(n)-1} \frac{1}{1-p(i)} \\
& \quad=\limsup _{\mu \rightarrow \infty} \sum_{j=3 \mu-1}^{3 \mu+2} \frac{29}{200} \prod_{i=\tau(j)}^{3 \mu-2} \frac{1}{1-29 / 200}=\frac{29}{200}
\end{aligned}
$$

$$
\begin{aligned}
& \cdot \limsup _{\mu \rightarrow \infty}\left\{\prod_{i=\tau(3 \mu-1)}^{3 \mu-2} \frac{1}{1-29 / 200}\right. \\
& +\prod_{i=\tau(3 \mu)}^{3 \mu-2} \frac{1}{1-29 / 200}+\prod_{i=\tau(3 \mu+1)}^{3 \mu-2} \frac{1}{1-29 / 200}
\end{aligned}
$$$$
\left.+\prod_{i=\tau(3 \mu+2)}^{3 \mu-2} \frac{1}{1-29 / 200}\right\}=\frac{29}{200}
$$$$
\text { - } \limsup _{\mu \rightarrow \infty}\left\{\prod_{i=3 \mu-5}^{3 \mu-2} \frac{1}{1-29 / 200}+\prod_{i=3 \mu-1}^{3 \mu-2} \frac{1}{1-29 / 200}\right.
$$$$
\left.+\prod_{i=3 \mu-1}^{3 \mu-2} \frac{1}{1-29 / 200}+\prod_{i=3 \mu-2}^{3 \mu-2} \frac{1}{1-29 / 200}\right\}=\frac{29}{200}
$$$$
\cdot \limsup _{\mu \rightarrow \infty}\left\{\left(\frac{1}{1-29 / 200}\right)^{4}+1+1+\frac{1}{1-29 / 200}\right\}
$$$$
\simeq 0.7309<1 \text {, }
$$$$
0.7309<1-\frac{1-\boldsymbol{\alpha}-\sqrt{1-2 \boldsymbol{\alpha}-\boldsymbol{\alpha}^{2}}}{2} \simeq 0.9875 \text {. }
$$

That is, conditions $\left(C_{1}^{\prime}\right),\left(C_{2}^{\prime}\right),\left(C_{5}^{\prime}\right),(52) \equiv(62)$ (for $\left.r=1\right)$ and $(55) \equiv(63)$ (for $r=1$ ) are not satisfied. 

(72).

Also, it is clear that condition $\left(C_{4}\right)$ is not applicable for

In addition, by using an algorithm on MATLAB software, we obtain

$$
\begin{gathered}
\limsup _{n \rightarrow \infty} \sum_{i=\sigma(n)}^{n} p(i) \prod_{j=\tau(i)}^{n} \frac{1}{1-P_{1}(j)} \simeq 4.6956 \\
<\frac{2}{1-\boldsymbol{\alpha}-\sqrt{1-2 \boldsymbol{\alpha}-\boldsymbol{\alpha}^{2}}} \simeq 80.1448
\end{gathered}
$$

which means that condition (71) is not satisfied.

The improvement of condition (68) over other conditions will be clear by comparing the values on the left-side of these conditions. For example, comparison with conditions $\left(C_{1}^{\prime}\right)$, $(52) \equiv(62)$ ( for $r=1$ ) and (65) (for $\ell=1$ ) gives percentage improvement of $76.7 \%, 40.21 \%$, and $20.15 \%$, respectively. Also, while conditions (62), (63), (65), and (66) do not lead to oscillation from the first iteration, condition (68) is satisfied from the first iteration, showing that, in our example, (68) is much faster than (62), (63), (65), and (66).

\section{Conclusion}

In the present paper we are concerned with the oscillatory behavior of linear delay difference equations with constant or time-varying argument. The problem is interesting since the corresponding first-order linear difference equation without delay does not possess oscillatory solutions when $p(n)<$ 1 and also by the fact that the mathematical modeling of many real-world problems lead to difference equations with retarded arguments. We present the most interesting sufficient oscillation conditions in the case of monotone or nonmonotone arguments and give examples which illustrate the applicability and the significance of the results.

\section{Conflicts of Interest}

The authors declare that there are no conflicts of interest regarding the publication of this paper.

\section{References}

[1] R. P. Agarwal, Difference Equations and Inequalities, Marcel Dekker, New York, NY, USA, 1992.

[2] R. P. Agarwal, M. Bohner, S. R. Grace, and D. O’Regan, Discrete oscillation theory, Hindawi Publishing Corporation, New York, 2005.

[3] R. P. Agarwal and P. J. Y. Wong, Advanced Topics in Difference Equations, vol. 404 of Mathematics and Its Applications, Kluwer Academic Publishers, Dordrecht, The Netherlands, 1997.

[4] P. G. Asteris and G. E. Chatzarakis, "Oscillation tests for difference equations with non-monotone arguments," Dynamics of Continuous, Discrete \& Impulsive Systems. Series A. Mathematical Analysis, vol. 24, no. 4, pp. 287-302, 2017.

[5] E. Braverman, G. E. Chatzarakis, and I. P. Stavroulakis, "Iterative oscillation tests for difference equations with several nonmonotone arguments," Journal of Difference Equations and Applications, vol. 21, no. 9, pp. 854-874, 2015.
[6] E. Braverman and B. Karpuz, "On oscillation of differential and difference equations with non-monotone delays," Applied Mathematics and Computation, vol. 218, no. 7, pp. 3880-3887, 2011.

[7] G. E. Chatzarakis, R. Koplatadze, and I. P. Stavroulakis, "Oscillation criteria of first order linear difference equations with delay argument," Nonlinear Analysis. Theory, Methods \& Applications. An International Multidisciplinary Journal, vol. 68, no. 4, pp. 994-1005, 2008.

[8] G. E. Chatzarakis, R. Koplatadze, and I. P. Stavroulakis, “Optimal oscillation criteria for first order difference equations with delay argument," Pacific Journal of Mathematics, vol. 235, no. 1, pp. 15-33, 2008.

[9] G. E. Chatzarakis, C. G. Philos, and I. P. Stavroulakis, "On the oscillation of the solutions to linear difference equations with variable delay," Electronic Journal of Differential Equations, vol. 2008, no. 50, pp. 1-15, 2008.

[10] G. E. Chatzarakis, C. G. Philos, and I. P. Stavroulakis, "An oscillation criterion for linear difference equations with general delay argument," Portugaliae Mathematica. A Journal of the Portuguese Mathematical Society, vol. 66, no. 4, pp. 513-533, 2009.

[11] G. E. Chatzarakis, I. K. Purnaras, and I. . Stavroulakis, "Oscillation of retarded difference equations with a non-monotone argument," Journal of Difference Equations and Applications, vol. 23, no. 8, pp. 1354-1377, 2017.

[12] G. E. Chatzarakis and L. Shaikhet, "Oscillation criteria for difference equations with non-monotone arguments," Advances in Difference Equations, Paper No. 62, 16 pages, 2017.

[13] M. P. Chen and Y. S. Yu, "Oscillations of delay difference equations with variable coefficients," in Proceedings of the Proc. First Intl. Conference on Difference Equations, pp. 105-114, 1995.

[14] S. S. Cheng and G. Zhang, "Virus in several discrete oscillation theorems," Applied Mathematics Letters, vol. 13, no. 3, pp. 9-13, 2000 .

[15] Y. I. Domshlak, "A discrete analogue of the Sturm comparison theorem for nonsymmetric equations," Dokl. Azerb. Acad. Sci, vol. 37 , no. 10 , pp. $12-15,1981$.

[16] Y. Domshlak, "Sturmian comparison method in oscillation study for discrete difference equations," Differential and Integral Equations: International Journal for Theory and Applications, vol. 7, no. 2, pp. 571-582, 1994.

[17] Y. Domshlak, "Delay-difference equations with periodic coefficients: sharp results in oscillation theory," Mathematical Inequalities \& Applications, vol. 1, no. 3, pp. 403-422, 1998.

[18] Y. Domshlak, "What should be a discrete version of the Chanturia-Koplatadze lemma?" Functional Differential Equations, vol. 6, no. 3-4, pp. 299-304, 1999.

[19] Y. Domshlak, "Riccati difference equations with almost periodic coefficients in the critical state," Dynamic Systems and Applications, vol. 8, no. 3-4, pp. 389-399, 1999.

[20] Y. Domshlak, "The Riccati difference equations near "extremal" critical states," Journal of Difference Equations and Applications, vol. 6, no. 4, pp. 387-416, 2000.

[21] L. H. Erbe and B. G. Zhang, "Oscillation of discrete analogues of delay equations," Differential and Integral Equations: International Journal for Theory and Applications, vol. 2, no. 3, pp. 300309, 1989.

[22] I. Gyori and G. Ladas, Oscillation Theory of Delay Differential Equations with Applications, Clarendon Press, Oxford, UK, 1991. 
[23] B. Karpuz, "Sharp oscillation and nonoscillation tests for linear difference equations," Journal of Difference Equations and Applications, vol. 23, no. 12, pp. 1929-1942, 2017.

[24] G. Ladas, "Recent developments in the oscillation of delay difference equations," in Proceedings of the International Conference on Differential Equations, Stability and Control, pp. 321-332, Dekker, New York, NY, USA, 1990.

[25] G. Ladas, C. G. Philos, and Y. G. Sficas, "Sharp conditions for the oscillation of delay difference equations," Journal of Applied Mathematics and Simulation, vol. 2, no. 2, pp. 101-111, 1989.

[26] B. S. Lalli and B. G. Zhang, "Oscillation of difference equations," Colloquium Mathematicum, vol. 65, no. 1, pp. 25-32, 1993.

[27] J. Shen and I. P. Stavroulakis, "Oscillation criteria for delay difference equations," Electronic Journal of Differential Equations, vol. 2001, no. 10, pp. 1-15, 2001.

[28] I. P. Stavroulakis, "Oscillations of delay difference equations," Computers \& Mathematics with Applications, vol. 29, no. 7, pp. 83-88, 1995.

[29] I. P. Stavroulakis, "Oscillation criteria for first order delay difference equations," Mediterranean Journal of Mathematics, vol. 1, no. 2, pp. 231-240, 2004.

[30] I. P. Stavroulakis, "Oscillation criteria for delay and difference equations with non-monotone arguments," Applied Mathematics and Computation, vol. 226, pp. 661-672, 2014.

[31] X. H. Tang, "Oscillations of delay difference equations with variable coefficients," J. Central So. Univ. of Technology, vol. 29, pp. 287-288, 1998.

[32] X. H. Tang and J. S. Yu, "Oscillation of delay difference equation," Computers \& Mathematics with Applications, vol. 37, no. 7, pp. 11-20, 1999.

[33] X. H. Tang and J. S. Yu, "A further result on the oscillation of delay difference equations," Computers \& Mathematics with Applications, vol. 38, no. 11-12, pp. 229-237, 1999.

[34] X. H. Tang and J. S. Yu, "Oscillations of delay difference equations," Hokkaido Mathematical Journal, vol. 29, no. 1, pp. 213-228, 2000.

[35] J. S. Yu, B. G. Zhang, and X. Z. Qian, "Oscillations of delay difference equations with oscillating coefficients," Journal of Mathematical Analysis and Applications, vol. 177, no. 2, Article ID 71267, pp. 432-444, 1993.

[36] J. S. Yu, B. G. Zhang, and Z. C. Wang, "Oscillation of delay difference equations," Applicable Analysis: An International Journal, vol. 53, no. 1-2, pp. 117-124, 1994.

[37] K. Gopalsamy, Stability and Oscillations in Delay Differential Equation of Population Dynamics, Kluwer Academic Publishers, 1992.

[38] G. S. Ladde, V. Lakshmikantham, and B. Z. Zhang, Oscillation Theory of Differential Equations with Deviating Arguments, Marcel Dekker, New York, NY, USA, 1987.

[39] V. Lakshmikantham and D. Trigiante, Theory of Difference Equation-Numerical Methods and Applications, Academic Press, Boston, Mass, USA, 1988. 


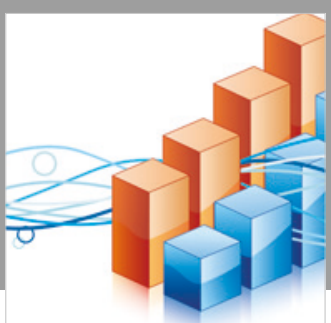

Advances in

Operations Research

\section{-n-m}
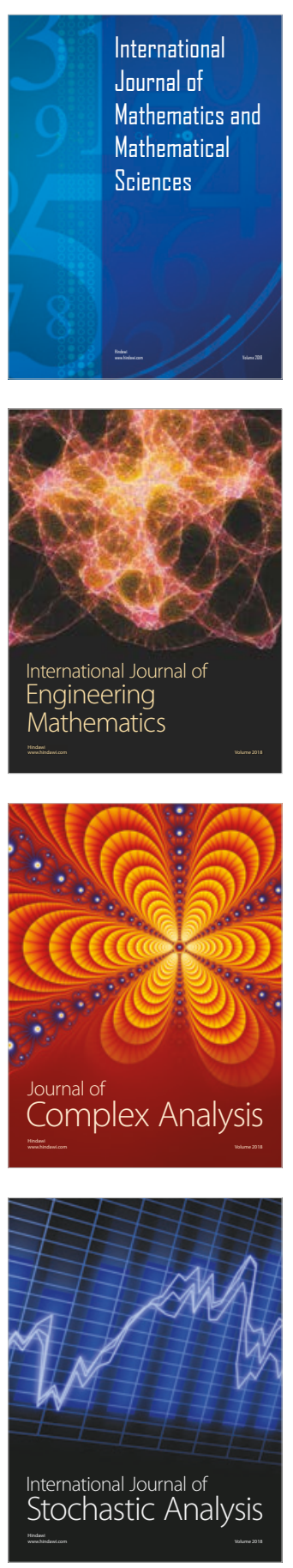
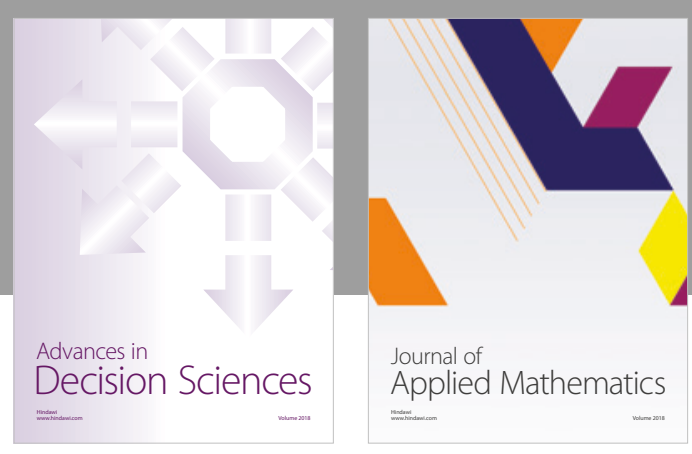

Journal of

Applied Mathematics
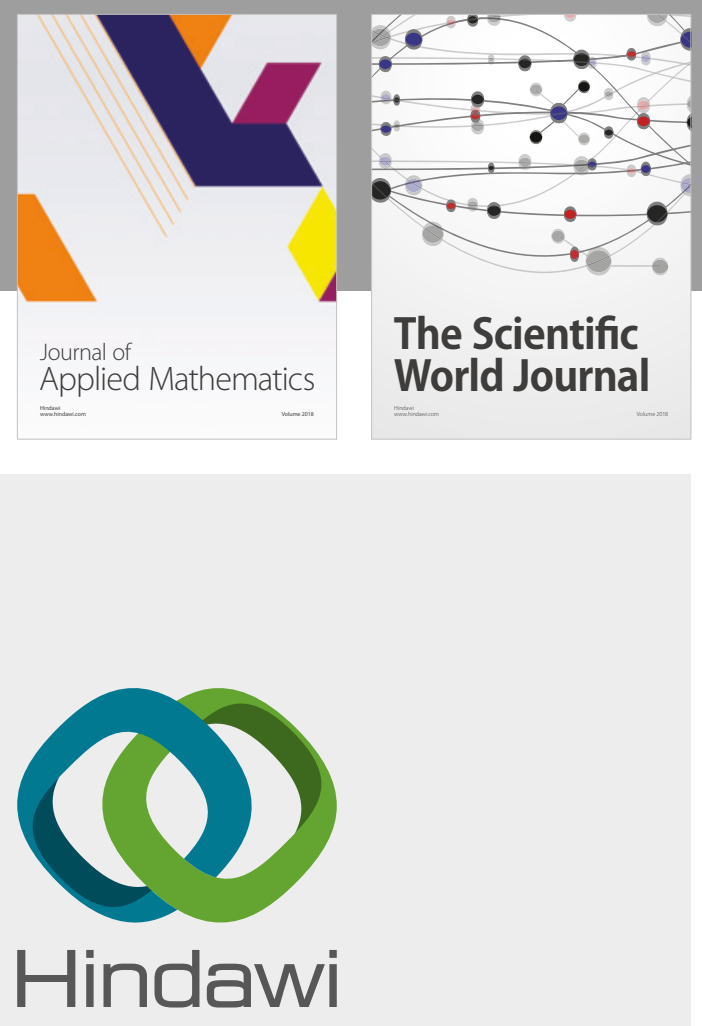

Submit your manuscripts at

www.hindawi.com

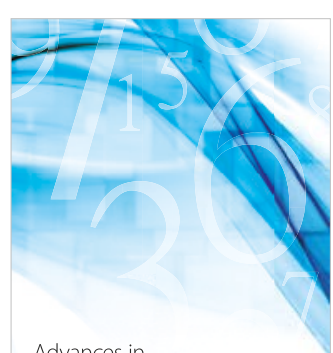

Advances in
Numerical Analysis
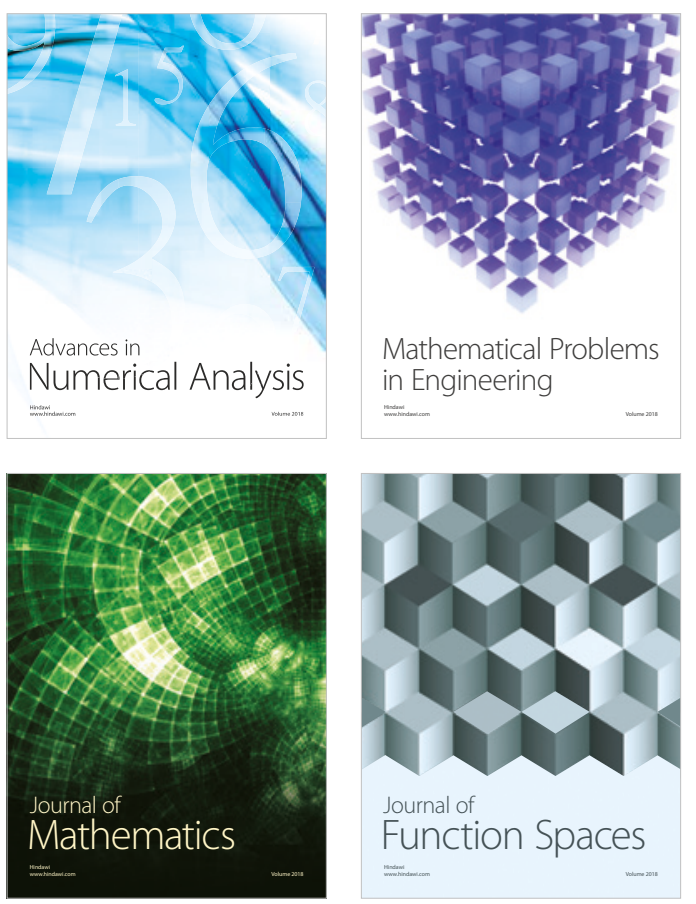

Mathematical Problems in Engineering

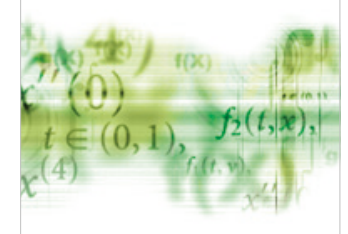

International Journal of

Differential Equations

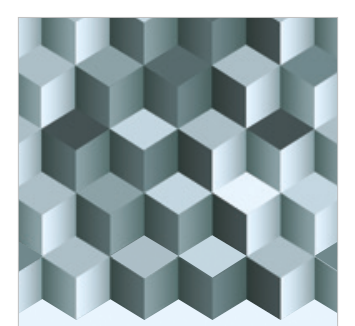

Journal of

Function Spaces

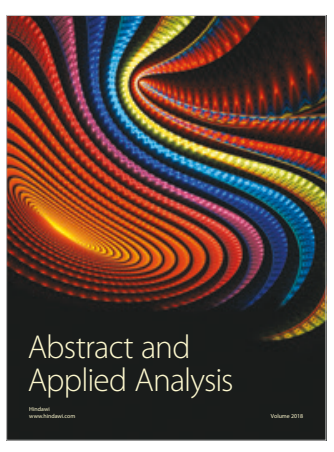

The Scientific

World Journal

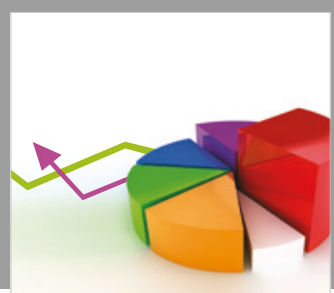

Journal of

Probability and Statistics
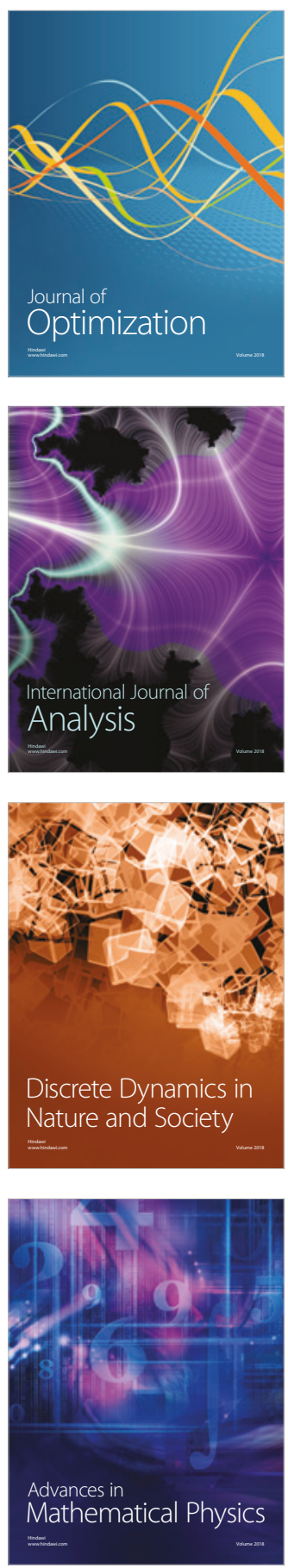